\title{
Bioavailability and dissolution of proprietary and generic formulations of phenytoin
}

\author{
Imad Soryal, Alan Richens
}

\begin{abstract}
A comparative study of the bioavailability of seven formulations of phenytoin was carried out on 17 patients with epilepsy who were taking phenytoin regularly as part of their drug therapy. Three patients withdrew for personal reasons. No significant differences were found between Epanutin capsules and other generic formulations. However significant differences were noted between the generic products. Phenytoin BP tablets manufactured by Regent Laboratories (now withdrawn) had a relative bioavailability of only $76 \%$ compared with tablets manufactured by $A$ H Cox and Company. In vitro dissolution tests requirements were met by all formulations of generic $100 \mathrm{mg}$ tablets, and it was concluded that in vitro dissolution tests are not reliable indicators of biological equivalence. Significantly higher plasma levels were found with Epanutin Infatabs, but this was accounted for by their higher content of phenytoin, which is present in the acid form rather than the sodium salt.
\end{abstract}

$(\mathcal{F}$ Neurol Neurosurg Psychiatry 1992;55:688-691)

Phenytoin remains one of the most commonly prescribed anticonvulsants despite its first introduction into clinical use over 50 years ago. It is, however, poorly water soluble and this property gives rise to inherent bioavailability problems. $^{12}$

The use of generic formulations has been encouraged because of concern about escalating health care costs. Before prescribing a generic product, however, the physician needs to be assured that the product is bioequivalent to proprietary formulations and other generic formulations. $^{3}$

The clinical relevance of inequivalence between formulations was first highlighted by an outbreak of phenytoin toxicity in the late 1960s in Australia. ${ }^{45}$ This was caused by a change of the excipient from calcium sulphate to lactose in a capsule formulation. Many other reports from Europe, North America and Australasia have described significant differences between preparations. These have included comparative single dose kinetic studies ${ }^{6-10}$ and investigations in steady state, some of which have suggested that such differences may have serious consequences in terms of therapeutic failure or intoxication. ${ }^{211-18}$
There have been three comparative studies in the UK dealing with the issue of the bioavailability of generic phenytoin tablets. Two described steady state plasma levels at one or two time points in epileptic patients, and gave conflicting results. ${ }^{1920}$ In both studies only a limited number of available generic formulations were used. The first of these two studies showed no difference between five generic formulations and Epanutin capsules ${ }^{19}$ while Hodges et $a l^{20}$ showed a significant difference between a single generic product and the propriety brand. The third study used a single dose technique in healthy volunteers. ${ }^{21}$ An intravenous dose was also given, enabling absolute bioavailability to be calculated; all formulations studied were found to be bioequivalent. At present in the UK solid oral formulations of phenytoin are available as "Epanutin" capsules and Infatabs, and as BP generic tablets produced by several different manufacturers. This study was instigated to reexamine the question of comparative bioavailability in steady state for all these formulations.

\section{Methods}

A single blind crossover study was designed consisting of seven four week treatment periods, each followed by a study day in our Epilepsy Unit where serial blood samples were collected for an interdose period of 12 hours. This study was undertaken in the first half of 1989 and all medications were obtained through the pharmacy at the University Hospital of Wales. The following phenytoin preparations were included:

Treatment A: Epanutin-Parke Davis $100 \mathrm{mg}$ capsules lot BN8G261

$50 \mathrm{mg}$ capsules lot BN8G449

Treatment B: Phenytoin BP-Evans Medical

$100 \mathrm{mg}$ tablets lot E70611A

$50 \mathrm{mg}$ tablets lot E70407B

Treatment C: Phenytoin BP-APS

$100 \mathrm{mg}$ tablets lot $71210 \mathrm{~A} / 2$

$50 \mathrm{mg}$ tablets lot $80937 \mathrm{~A} / 1$

Treatment D: Infatabs*_Parke Davis $50 \mathrm{mg}$ tablets lot BN8G255

Treatment E: Phenytoin BP-A H Cox $100 \mathrm{mg}$ tablets lot PY37

$50 \mathrm{mg}$ tablets lot PY12 
Treatment F: Phenytoin BP-Thomas

Kerfoot

$100 \mathrm{mg}$ tablets lot KN21B2

$50 \mathrm{mg}$ tablets lot KB54A

Treatment G: Phenytoin BP†-Regent Laboratories

$100 \mathrm{mg}$ tablets lot A073373AB

$50 \mathrm{mg}$ tablets lot A072086BB

*Epanutin Infatabs contain $50 \mathrm{mg}$ of phenytoin acid. All the other solid formulations contain the sodium salt. $50 \mathrm{mg}$ of phenytoin acid $\equiv$ (equivalent to) $54 \mathrm{mg}$ of sodium salt. tThe Regent laboratories preparation was withdrawn from the market in 1989 because of technical difficulties with a new formulation that incorporated different excipients and had poor bioavailability during in-house testing.

Seventeen patients regularly attending the Epilepsy Unit took part, 11 women and 6 men. Their ages varied between 18 and 67 years and they were all within $\pm 20 \%$ of their ideal body weight. All were on maintenance treatment with phenytoin only in or in combination with one or more other anticonvulsants. The daily dose varied from 200-500 mg between patients, but for each individual the doses of all drugs were kept unaltered throughout the study. The sequence of formulations was determined randomly, and was kept undisclosed to monitoring clinicians. Daily doses were standardised for all patients into twice daily doses (12 hourly) and compliance was checked by tablet counts and dose diaries. The study was approved by the local Ethics Committee.

Blood samples were collected predose and hourly for 12 hours. Plasma was separated by centrifugation and was frozen at $-20^{\circ} \mathrm{C}$ until analysis. Phenytoin concentration was measured by an in house HPLC method. The coefficient of variation of the assay was $<4 \%$ at levels of $5-20 \mathrm{mg} / \mathrm{l}$. Calibration curves for phenytoin were constructed daily during the course of these studies. All curves afforded a correlation coefficient of 0.999 or better.

The following pharmacokinetic parameters were calculated from the raw data:

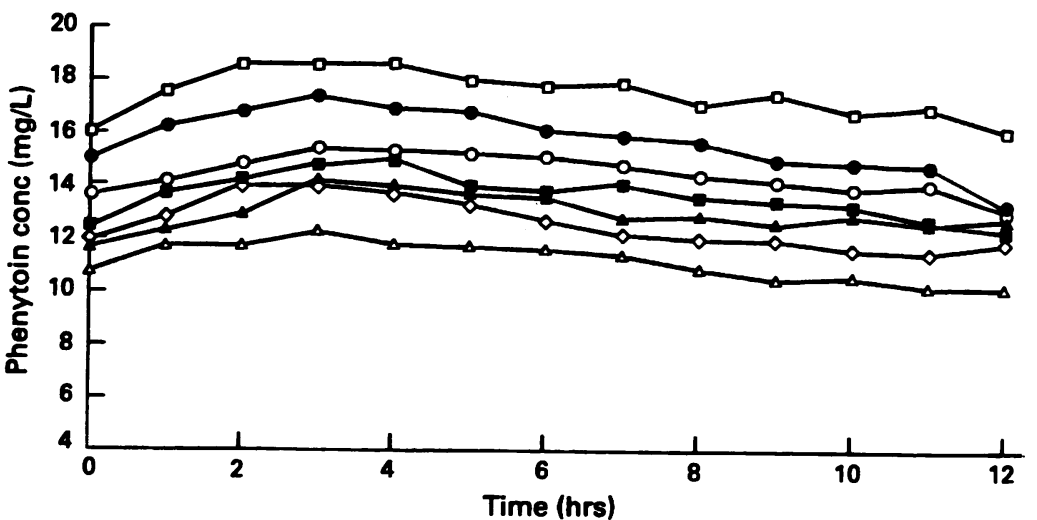

- Epanut (PD) - Evans tab - APS tab a Infatabs (PD)

- AH Cox tab $\diamond$ T Kerfoot $\triangle$ Regents lab tab

Figure Mean plasma phenytoin concentration over a 12 hour interdose interval in 14 patients who received each of the seven phenytoin formulations for 4 weeks.
$C$ max-Observed maximum plasma concentration $(\mathrm{mg} / \mathrm{l})$ (extracted directly from raw data)

$T$ max-Observed time to maximum concentration (h) (extracted directly from raw data)

$A U C$ 0-12 Area under the plasma phenytoin concentration/time curve up to 12 hours (mg. $\left.1^{-1} \cdot h\right)$. This was calculated using the independent model free trapezoidal rule.

Fluctuation This assessed the percentage variation from maximum to minimum concentration and was calculated according to the following formula:

$$
\text { Fluctuation }=\frac{\mathrm{C}_{\max }-\mathrm{C}_{\min } \times 100 \%}{\mathrm{C}_{\min }}
$$

$\mathrm{C}_{\min }$ and $\mathrm{C}_{\max }$ were extracted directly from raw data.

Statistical analysis Two way analysis of variance was used to compare differences between patients and between the seven formulations. This was performed using the SPSS ANOVA package. At all times a level of $p<0.05$ was taken as the minimum for statistical significance. Any significant differences between treatments or formulations derived from this were investigated further using range tests (Student-Newman-Keuls procedure) to identify where the differences lay. Friedman two way ANOVA was also used to compare differences in seizure frequency, incidence of side effects and percentage fluctuation.

Seizure frequency Seizures were recorded by the patients on a seizure chart used routinely in the Epilepsy Unit.

Side effects All unwanted effects spontaneously reported, observed, or elicited by direct questioning or indirectly were recorded.

Dissolution procedure Dissolution was studied using the standard paddle method and repeated for the Epanutin capsules using the basket method. $^{22}$ The medium was water and the volume $1000 \mathrm{ml}$. Only $100 \mathrm{mg}$ tablets/capsules were studied. Determination of the amount of phenytoin dissolved was by ultraviolet absorbance at 310 to $320 \mathrm{~nm}$ of filtered portions of the solution under test using as a comparison a known concentration of phenytoin in the same medium.

\section{Results}

Fourteen patients completed the trial, two withdrew for personal reasons and one because of pregnancy. One patient repeated a whole leg because of error in the understanding of the correct dose. The overall compliance was good, being greater than $90 \%$ in all treatments.

Plasma phenytoin concentrations The profile for mean plasma phenytoin concentrations for sampling times during different treatments is shown in the figure. 
Table 1 Mean (SD) Cmax and AUC 0-12 for each phenytoin formulation. The relative bioavailability in comparison with Epanutin capsules has been calculated

\begin{tabular}{|c|c|c|c|}
\hline Formulation & $\begin{array}{l}\text { Cmax mean } \\
(1 S D)(m g / l)\end{array}$ & $\begin{array}{l}A U C \text { mean } \\
(1 S D)\left(m g . l^{-1} \cdot h\right)\end{array}$ & $\begin{array}{l}\text { Relative } \\
\text { Bioavailability \% }\end{array}$ \\
\hline $\begin{array}{l}\text { Epanutin capsules } \\
\text { Phenytoin BP Evans Medical } \\
\text { Phenytoin BP APS } \\
\text { Epanutin Infatabs } \\
\text { Phenytoin BP A H Cox } \\
\text { Phenytoin BP Thomas Kerfoot } \\
\text { Phenytoin BP Regent Laboratories }\end{array}$ & $\begin{array}{l}16 \cdot 4(5 \cdot 7) \\
15 \cdot 1(5 \cdot 5) \\
15 \cdot 6(5 \cdot 5) \\
20 \cdot 0(7 \cdot 9) \\
18 \cdot 2(7 \cdot 6) \\
15 \cdot 1(5 \cdot 4) \\
13 \cdot 1(4 \cdot 2)\end{array}$ & $\begin{array}{l}174.6(58.8) \\
155.9(55.6) \\
160.7(53.7) \\
210.7(83.9) \\
187.5(82.8) \\
148.2(60.9) \\
132.8(44.4)\end{array}$ & $\begin{array}{r}100 \\
92 \\
92 \\
121 \\
107 \\
85 \\
76\end{array}$ \\
\hline
\end{tabular}

$C$ max: The mean $C \max$ for the different preparations is shown in table I. Statistically significant differences were identified on analysis of variance between formulations $(p<0.001)$ and patients $(p<0.001)$. Range tests showed that Epanutin Infatabs differed significantly from four of the generic formulations (Evans, APS, Kerfoot, and Regent). The Regent generic tablet also differed significantly from the Cox generic tablet. It was clear that the formulation with the lowest Cmax differed significantly from the two formulations with the highest Cmax.

$T$ max Analysis of mean T-max for the seven different treatments fell short of achieving statistical significance $(p<0.066)$. There were also no significant differences between patients.

AUC $0-12$ Mean AUC for the different formulations is given in table 1 . Statistically significant differences were seen between formulations on analysis of variance $(p<0.001)$. Epanutin Infatabs had a significantly higher AUC than the Evans, APS, Kerfoot and Regent tablets. The latter formulation also had an AUC significantly less than the Cox tablet. For comparative purposes, Epanutin capsules were taken as $100 \%$ bioavailable and the relative bioavailability of the other formulations was calculated (table 1). An analysis of the AUC values we also performed using confidence intervals, and the results are given in table 2. The results are in close agreement with the analysis of variance.

Fluctuation No significant differences were seen between formulations but differences between patients were significant $(p<0.02)$.

Side effects and seizure frequency No statistically significant differences were found when comparing the incidence of side effects and seizure frequency during the seven treatments. The side effects commonly experienced were

Table 2 Confidence intervals comparing the different phenytoin formulations

\begin{tabular}{lll}
\hline Phenytoin formulation & Confidence interval & $p$ \\
\hline Epanutin vs Evans & $(-26,63)$ & 0.41 \\
Epanutin vs APS & $(-30,58)$ & 0.51 \\
Epanutin vs Infatabs & $(-93,20)$ & 0.2 \\
Epanutin vs A H Cox & $(-69,43)$ & 0.64 \\
Epanutin vs Kerfoot & $(-20,73)$ & 0.25 \\
Epanutin vs Regent & $(-1,82)$ & 0.044 \\
Infatabs vs Evans & $(-5,110)$ & 0.055 \\
Infatabs vs APS & $(5,120)$ & 0.073 \\
Infatabs vs Kerfoot & $(25,131)$ & 0.034 \\
Infatabs vs Regent & $(2,107)$ & 0.006 \\
Cox vs Regent & ( & 0.042 \\
\hline
\end{tabular}

headaches, drowsiness, visual disturbance, mental slowness, fatigue and malaise. Preferences of individual patients to particular formulations varied widely.

Dissolution tests The mean time taken for $70 \%$ of active drug to dissolve from the generic formulations varied from 13 minutes for the Regent generic to 8 minutes for the Cox generic. The mean time for Epanutin capsules was 54 minutes (paddle method) and 25 minutes (basket method). The official British Pharmacopoeia dissolution test requirement for phenytoin is $70 \%$ dissolution within 45 minutes. All the tablets were well within this requirement and the difference between them was not significant. The dissolution times for Epanutin capsules cannot be compared with the generic tablets because the active constituents may adhere to the gelatine capsules. However, it was notable that capsules varied greatly from one another in their rate of dissolution compared to other tablets. This agrees with previous dissolution studies. ${ }^{20}$

\section{Discussion}

Comparative bioavailability studies of antiepileptic drugs can be performed either in healthy volunteers given single doses or in epileptic patients who are receiving the drug regularly and are in steady state. The latter technique has the advantage of greater sensitivity because plasma levels are higher (and therefore assay precision is greater) and also, for phenytoin, because the saturation kinetics exhibited by this drug ${ }^{1}$ exaggerate bioavailability differences. Furthermore, it allows real differences between formulations to be assessed under clinical conditions. The object of this study was to examine the comparative bioavailability of all formulation of phenytoin marketed in the UK. The results indicate that there are clinically significant differences between generic formulations.

Epanutin capsules did not differ from the generic formulations but Epanutin Infatabs differed significantly from four of the other formulations in Cmax and AUC 0-12. The explanation for this is that Infatabs contain $50 \mathrm{mg}$ of phenytoin in the acid form, whereas all other formulations contain the sodium salt. Infatabs therefore contain about $8 \%$ more phenytoin than an equivalent $50 \mathrm{mg}$ Epanutin capsule or generic tablet. This appears not to be widely appreciated by the prescriber, but a clinically significant change in phenytoin level can result from a change from Infatabs to another formulation. Stewart et $a l^{14}$ found a similar difference in their study but did not appreciate at that time that the phenytoin content of Infatabs and generic tablets differed and therefore concluded that a bioavailability difference was present between the two.

Two generic formulations differed significantly from each other in $\mathrm{Cmax}$ and AUC. The Cox tablet had the greatest AUC of all the formulations studied while the Regent tablet had the smallest. The magnitude of the differences found was such that a marked change in steady state phenytoin concentration would be 
expected in changing from one formulation to the other. Substitution of the Regent tablet for the Cox tablet might result in an increase in seizure frequency, while a reverse change might precipitate phenytoin intoxication. This danger, however, no longer exists because the Regent generic was withdrawn in 1989 following technical difficulties. Changes between the other generic formulations studied would have a smaller effect which would be unlikely to have clinical consequences. The absence of a significant difference in T-max suggests that the Regent and Cox formulations differed mainly in the extent rather than the rate of absorption.

No significant differences were seen in seizure control during the seven different treatments. This is probably due to the fact that a four week treatment period is too short for assessment of efficacy of therapy. However, this was not the primary aim of the study.

We conclude that substitution of one generic formulation of phenytoin for another or for Epanutin capsules in the UK may be associated with a change in bioavailability of a magnitude that is likely to cause changes in seizure control or incidence of adverse effects. In-house testing in the manufacturing company using in vitro techniques may not be sensitive enough to detect clinically-important differences in vivo. Although in the case of the Regent tablet, deficiencies were detected inhouse, and led to withdrawal of the formulation, we believe that wider use should be made of in vivo bioavailability testing following any variation in the manufacturing process. In vitro tests may, however, be adequate for batch to batch quality control.

The British National Formulary warns that different brands of phenytoin tablets may not be interchangeable with one another. This warning should be heeded by clinicians who prescribe phenytoin and by pharmacists who stock and dispense the drug.
1 Richens A. Clinical pharmacology of phenytoin. Clin Pharmacokinet 1979;4:153-69.

2 Neuvonen PJ, Brady A, Lehtovaara R. Effect of increased bioavailability of phenytoin tablets on serum phenytoin bioavailability of phenytoin tablets on serum phenytoin
concentration in epileptic outpatients. $B r \mathcal{F}$ Pharmacol concentration

3 Nuwer MR, Browne TR, Dodson WE, et al. Generic substitutions for antiepileptic drugs. Neurology

4 Tyrer JH, Eadie MJ, Sutherland JM, Hooper WD. Outbreak of anticonvulsant intoxication in an Australian city. $B M \mathcal{F}$ 1970;4:271-3

5 Bochner R, Hooper WD, Tyrer JH, Eadie MJ. Factors involved in an outbreak of phenytoin intoxication. $\mathcal{f}$ Neurol Sci 1972;16:481-7.

6 Martin CM, Rubin M, O'Malley W, Garagnia VF, MacCauley CE. Comparative physiological availability of "brand" and "generic" drugs in man: chloramphenicol, sulfisoxazole and diphenylhydantoin. Pharmacologist 1968;10:167.

7 Lund L. Clinical significance of generic inequivalence of three different pharmaceutical preparations of phenytoin. Eur f Clin Pharmacol 1974;7:119-24.

8 Pentikainen PJ, Neuvonen PJ, Elfving SM. Bioavailability of four brands of phenytoin tablets. Eur $\mathcal{f}$ Clin Pharmacol 1975;9:213-18.

9 Gugler R, Manion CV, Azarnoff DL. Phenytoin: pharmacokinetics and bioavailability. Clin Pharmacol Ther 1976;19:135-42.

10 Neuvonen PJ, Pentikainen PJ, Elfving SM. Factors affecting bioavailability of phenytoin. Int $\mathcal{7}$ Clin Pharmacol Biopharmacol 1977;15:84-89.

11 Appleton DB, Eadie MJ, Hooper WD, Lucas B, Sutherland JM, Tyrer JH. Blood phenytoin concentrations produced by ingestion of three different phenytoin preparations. Med $\Im$ Aust 1972;1:410-12.

12 Sansom LH, O'Reilly WJ, Wiseman CW, Sterr LM, Dreham J. Plasma phenytoin levels produced by various phenytoin preparations. Med $\mathcal{f}$ Aust 1975;2:593-5.

13 Manson JI, Beal SM, Magarey A, Pollard AG, O'Reilly WJ. Bioavailability of phenytoin from various pharmaceutical preparations in children. Med $\mathcal{F}$ Aust 1975;2:590-2.

14 Stewart MJ, Ballinger BR, Devlin EJ, Miller AY, Ramsay AC. Bioavailability of phenytoin. A comparison of two preparations. Eur $₹$ Clin Pharmacol 1975;9:209-12.

15 Richens A. Drug Treatment of Epilepsy. London, Henry Kimpton 1976.

6 Tammisto P, Kanto K, Viudari M. Bioavailability of phenytoin. Lancet 1976;1:154-255.

17 Rambeck B, Boenigk HE, Stenzel E. Bioavailability of three phenytoin preparations in healthy subjects and in epileptics. Eur f Clin Pharmacol 1977;12:285-90.

18 Takahashi K, Honda J, Takahashi H, Ohtsuka C. Diphenylhydantoin blood levels in childhood epilepsy: a comparison of two preparations with different particle size. Brain Dev (Tokyo) 1979;11:278-84.

9 Chen S, Allen K, Oxley J, Richens A. Comparative bioavailability of phenytoin from generic formulations in the United Kingdom. Epilepsia 1982;23:149-52.

20 Hodges S, Forsythe WI, Gillies D, Remington H, Cawood A. Bioavailability and dissolution of three phenytoin preparations for children. Develop Med Child Neurol 1986;28:708-12.

21 Hirii MR, Measuria H, Kuhn S, Mucklow JC. A comparative study of the bioavailability of five different phenytoin preparations. $₹$ Pharm Pharmacol 1985;37:570-2.

22 British Pharmacopoeia 1988. Appendix XII, Al43-4. 